\title{
Effect of shortened Integrated Management of Childhood Illness training on classification and treatment of under-five children seeking care in Rwanda
}

\author{
This article was published in the following Dove Press journal: \\ Risk Management and Healthcare Policy \\ 15 May 2014 \\ Number of times this article has been viewed
}

Jean-Modeste Harerimana' Laetitia Nyirazinyoye' Jean-Bosco Ahoranayezu²

Ferdinand Bikorimana ${ }^{3}$

Bethany L Hedt-Gauthier ${ }^{1,4}$ Katherine A Muldoon ${ }^{5}$

Edward J Mills ${ }^{6,7}$

Joseph Ntaganira'

'University of Rwanda College of Medicine and Health Sciences School of Public Health, Kigali, Rwanda; ${ }^{2}$ Community Vision Initiative, Kigali, Rwanda; ${ }^{3}$ Maternal and Child Health, Child Unit, Rwandan Ministry of Health, Kigali, Rwanda; ${ }^{4}$ Harvard Medical School, Boston, MA, USA; ${ }^{5}$ University of British Columbia, Vancouver, BC, Canada; ${ }^{6}$ University of Ottawa, Ottawa, ON, Canada; ${ }^{7}$ Stanford University, Stanford, CA, USA
Correspondence: Jean-Modeste Harerimana

University of Rwanda College of Medicine and Health Sciences School of Public Health, PO Box 3286, Kigali, Rwanda Email modesteharera@gmail.com
Background: Integrated Management of Childhood Illness (IMCI) is an effective 11-day standard training; however, due to budgetary expenses and human resource constraints, many health professionals cannot take 11 days off work. As a result, shortened training curriculums (6-day) have been proposed. We used a cross-sectional study to evaluate the effect of this shortened training on appropriate IMCI classification and treatment of under-five childhood illness management in Rwanda.

Methods: A cross-sectional study was conducted in 22 health centers in Rwanda, comparing data from 121 nurses, where 55 nurses completed the 11-day and 66 nurses completed the 6-day training. Among 768 children, we evaluated clinical outcomes from May 2011 to April 2012. Descriptive statistics were used to display the sociodemographic characteristics of health providers; including level of education, sex, age, and professional experiences. Bivariable and multivariable analyses were used to test for differences between nurses in the 6-day versus 11-day training on the appropriate classification and treatment of childhood illness.

Results: Our findings show that at the bivariable level and after controlling for confounders in the multivariable analysis, the only significant differences detected between nurses in the long and short training was the classification of fever (adjusted odds ratio [aOR] 0.7, 95\% confidence interval [CI] 0.64-0.75) and treatment of pneumonia (aOR 0.8, 95\% CI 0.70-0.89). Nurses in the short training had lower odds of inappropriate misclassification and treatment for these two conditions.

Conclusion: There was no difference in classification and treatment of childhood illness among nurses who completed the standard and short IMCI training courses. Short-training could be a more cost-saving option for health facilities without compromising the key outcomes related to case management.

Keywords: IMCI, child health, cost-saving

\section{Background}

Integrated Management of Childhood Illness (IMCI) is a strategy, developed in 1996 by the World Health Organization (WHO) and United Nations Children's Fund (UNICEF), ${ }^{1,2}$ designed to improve child health and reduce under-five mortality in poor countries. $^{3}$ The IMCI program is a WHO program with three components designed to improve the wellbeing of children under 5 years of age: improving health providers' skills, strengthening health systems, and engaging community to improve family practices. $^{4,5}$ 
The global under-five mortality rate has declined from 87 deaths per 1,000 live births in 1990 to 51 in $2011 .^{2}$ Still, nearly 6.9 million children will die annually before reaching the age of 5 years, and the majority of these deaths occur in countries with limited resources. ${ }^{2}$ In sub-Saharan Africa, the infant mortality rate (children $<12$ months) is one death per nine live births; this is more than 16 times the average for developed regions (one death per 152 live births). ${ }^{2}$

To address the inequitable global dispersion of infant and child mortality, the IMCI strategy was developed based on evidence from health workers serving populations with high mortality rates, and addresses common causes of death mainly attributed to malaria, pneumonia, diarrhea, measles, human immunodeficiency virus (HIV), and malnutrition in developing countries. ${ }^{6,7}$ Malaria and pneumonia contribute to almost $40 \%$ of the under-five mortality in subSaharan-Africa. ${ }^{8}$ The IMCI program trains health providers in the appropriate administration of medicine, counseling on nutritional feeding, home treatment, and effective reassessment of sick children. ${ }^{9}$ Providers are trained to identify signs and symptoms of common childhood illness, nutritional status, and immunization status of the child, classification of illnesses, and appropriate treatment. ${ }^{10}$

Globally, the WHO IMCI standard training (also called long training) is an in-service training using IMCI materials lasting 11 days and combines classroom work with clinical practice. ${ }^{9,11}$ Since 2001, IMCI national and international focal persons have reported to WHO that access to quality IMCI service is limited by budget, staff turnover, and having few facilitators. ${ }^{3}$ Concerns have been raised in many countries that the 11-day standard training is too expensive and has implications on the delivery of services at health facilities, since staff need to be absent from their other duties for long training periods. ${ }^{3,12}$ Evaluations of the IMCI training have been conducted in Zambia and Kosovo and show preliminary results that suggest that a shortened training may not reduce the clinical classification and management of childhood illnesses; ${ }^{9,11}$ however, more evidence is needed.

Rwanda is classified among the countries in the world with high early childhood mortality, where the infant mortality rate is more than 40 deaths per 1,000 live births. ${ }^{13} \mathrm{In}$ Rwanda, one out of every 13 children dies before their fifth birthday, approximately 140,000 children per year, with an under-five mortality rate estimated at 76 per 1,000 live births..$^{14}$ Over $66 \%$ of these deaths occur within the first year of life, as estimated by an infant mortality of 50 per 1,000 live births, and 27 neonatal deaths per 1,000 live births occur in the first month of life, representing about $54 \%$ of the infant mortality and $35 \%$ of the under-five mortality. ${ }^{14}$ The IMCI strategy was officially adopted by the Rwandan Government Ministry of Health in 2000 as a priority intervention to reduce infant and child mortality, but the establishment of an IMCI working group and implementation of the program did not start for several years. ${ }^{15}$

Since 2008, the IMCI training course has proven to be expensive for the Ministry of Health and its partners in terms of training costs. ${ }^{15}$ Additionally, it has taken health workers away from their health facilities for long periods at a time while they participate in the training. In 2011, one of Rwanda's strategies was to shorten the IMCI training from 11 days to 6 days, decreasing the cost of training from an average of US $\$ 1,000$ to approximately US\$600 per trainee..$^{15}$ While this change was cost-saving, there is currently no evaluation on the effect of the shortened IMCI training course on nurses' performance (knowledge and skills acquired during training) in Rwanda. The objective of this study was to evaluate the effect of shortened training versus the standard training on the appropriate classification and treatment of under-five illnesses management.

\section{Methods}

\section{Setting}

Our study includes two districts, Nyanza and Ruhango, out of 30 districts in Rwanda. This study was a cross-sectional study carried out between May and July 2012, in 22 health centers. In eleven health centers in Ruhango district, the nurses were trained for 6 days (short training), and in eleven health centers in Nyanza district, staff were trained for the standard 11 days (long training). All eligible health centers in the district were included in the study. A health center in Nyanza district was eligible if there were at least two nurses trained for a standard training and had more than 1 year of IMCI implementation. A health center in Ruhango district was eligible if it had at least two nurses trained in the shortened IMCI training and had at least 1 year of IMCI implementation.

\section{Data sources and variables of interest}

Data were collected from the administrative medical records of sick children from each health center. Variables of interest included individual characteristics of the nurses who participated in each training and included level of nursing training (registered nurses, nurses who has graduated from a nursing program at a post-secondary institute or from nursing school; or associate nurses, nurses who completed a 3 -year nursing program in secondary school), sex, age group 
( $<35$ versus $\geq 35$ years old), and length of time in the work force ( $<2$ versus $\geq 2$ years).

The main outcome of interest was the appropriate classification and treatment of six common under-five childhood illnesses: pneumonia, diarrhea, fever, ear infection, management of HIV, and malnutrition. Using the data collected on the signs and symptoms, study team members extracted data on the nurses' classification of childhood illness and treatment provided. Referring to the main symptoms and the IMCI chart booklet, the study IMCI expert compared the signs and symptoms against the classification and treatment given by the nurses and confirmed whether the classifications and treatment were appropriate or inappropriate. The appropriate classification was defined as agreement between documented classification and the classification performed by study staff based on the symptoms recorded in the sick child's medical record and IMCI guideline. Appropriate treatment was defined as the child being prescribed the correct drug in the correct dosage based on the Rwanda adaptation of the IMCI case management guidelines. The key outcome of interest was the appropriate classification and treatment of each illness.

\section{Data analysis}

Descriptive statistics including frequencies and proportions were used to display the characteristics of the sampled nurses. A chi-squared test was used to test for statistical significant differences in both individual characteristics of nurses who completed the long versus short training course and differences in the appropriate classification and treatment of six childhood illnesses.

We ran 12 individual bivariate logistic regression models to determine the odds of appropriate classification and treatment of the six childhood illnesses among nurses who completed the short training course compared with nurses who completed the long course. We then ran 12 multivariable logistic regression models to assess these associations while controlling for confounding variables including age, sex, and length of time in the workforce. All analyses were completed using Stata VII (StataCorp, College Station, TX, USA). ${ }^{16}$

\section{Ethics statement}

The National IMCI coordinator and the leaders of each health center approved this study before data collection. The National University of Rwanda School of Public Health Research Consultancy and Technology Transfer committee additionally approved this study.

\section{Results}

Table 1 displays the sociodemographic characteristics of 121 nurses included in this study. Between the short 6-day and long 11-day training, there were statistically significant differences for level of training and sex. Among the 66 nurses in the short training, $53(80.3 \%)$ were associate nurses compared with $53(96.4 \%)$ of the 55 nurses in the long training $(P=0.001)$. Of the 66 nurses in the short training, $37(56.0 \%)$ were female nurses compared with the group of 55 nurses in the long training, where $37(67.3 \%)$ were female nurses $(P=0.001)$. There were no significant differences in age group or length of time in the work force. From the total of 121 nurses, $81.0 \%$ of nurses were $<35$ years of age, and out of 121 nurses, 105 (86.6\%) had been working for $\geq 2$ years.

Table 2 displays the appropriate classification and treatment of childhood illnesses by comparing nurses who completed the long training to those who completed the short training. Significant differences were detected for the classification of fever, with significantly more children with fever being appropriately classified by nurses in the short training (88.3\%) compared with the long training (47.4\%) ( $P=0.001)$.

Marginally significant differences were detected for the appropriate classification of diarrhea, with more children being appropriately classified by nurses in the short training (85.0\%) compared with the long training $(74.3 \%)(P=0.053)$, and $77.1 \%$ of malnutrition cases seen by nurses in the long training were correctly classified compared with $71.2 \%$ of cases seen by nurses in the short training $(P=0.063)$. Nurses in the long training appropriately treated $62.0 \%$ of pneumonia diagnoses compared with $89.3 \%$ treated by nurses in the short training $(P=0.001)$. Nurses in the long training appropriately

Table I Sociodemographic characteristics of nurses $(n=|2|)$ included in this study

\begin{tabular}{|c|c|c|c|c|}
\hline $\begin{array}{l}\text { Demographic } \\
\text { characteristic }\end{array}$ & $\begin{array}{l}\text { Long } \\
\text { training } \\
(n=55)\end{array}$ & $\begin{array}{l}\text { Short } \\
\text { training } \\
(n=66)\end{array}$ & $\begin{array}{l}\text { Total } \\
(n=12 I)\end{array}$ & $P$-value \\
\hline \multicolumn{5}{|l|}{ Level of training } \\
\hline Registered nurse & $2(3.6 \%)$ & $13(19.7 \%)$ & 15 (I2.4\%) & 0.001 \\
\hline Associate nurse & $53(96.4 \%)$ & $53(80.3 \%)$ & $106(87.6 \%)$ & \\
\hline \multicolumn{5}{|l|}{ Sex } \\
\hline Male & $18(32.7 \%)$ & 29 (44.0\%) & 47 (38.8\%) & 0.001 \\
\hline Female & $37(67.3 \%)$ & 37 (56.0\%) & $74(61.2 \%)$ & \\
\hline \multicolumn{5}{|l|}{ Age } \\
\hline$<35$ years & $43(78.2 \%)$ & $55(82.8 \%)$ & $98(81.0 \%)$ & 0.168 \\
\hline$\geq 35$ years & $12(21.8 \%)$ & II (I7.2\%) & $23(19.0 \%)$ & \\
\hline \multicolumn{5}{|c|}{ Length of time in the workforce } \\
\hline$<2$ years & 7 (12.2\%) & $9(13.7 \%)$ & $16(13.2 \%)$ & 0.593 \\
\hline$\geq 2$ years & 48 (87.8\%) & 57 (86.3\%) & 105 (86.8\%) & \\
\hline
\end{tabular}


Table 2 Appropriate classification and treatment of common under-five childhood illnesses

\begin{tabular}{|c|c|c|c|c|c|}
\hline \multirow[t]{2}{*}{ Outcome } & \multicolumn{2}{|l|}{ Long training } & \multicolumn{2}{|l|}{ Short training } & \multirow[t]{2}{*}{$P$-value } \\
\hline & Appropriate & Total & Appropriate & Total & \\
\hline \multicolumn{6}{|l|}{ Classification/diagnoses } \\
\hline Pneumonia & 247 (91.4\%) & 270 & 278 (93.0\%) & 299 & 0.513 \\
\hline Diarrhea & $90(74.3 \%)$ & 121 & $94(85.0 \%)$ & 111 & 0.053 \\
\hline Fever & $122(47.4 \%)$ & 257 & $228(88.3 \%)$ & 258 & 0.001 \\
\hline Ear infection & 48 (62.0\%) & 78 & $4 \mid(68.3 \%)$ & 60 & $0.44 I$ \\
\hline HIV & $278(72.4 \%)$ & 384 & $29 \mid(75.8 \%)$ & 384 & 0.284 \\
\hline Malnutrition & $294(77.1 \%)$ & 381 & $274(71.2 \%)$ & 383 & 0.063 \\
\hline \multicolumn{6}{|l|}{ Treatment/management } \\
\hline Pneumonia & 67 (62.0\%) & 108 & II 8 (89.3\%) & 132 & 0.001 \\
\hline Diarrhea & 50 (75.7\%) & 66 & $48(84.2 \%)$ & 57 & 0.025 \\
\hline Fever (malaria) & $4(14.8 \%)$ & 27 & $5(29.4 \%)$ & 17 & 0.242 \\
\hline Ear infection & 4 (44.4\%) & 9 & $2(33.3 \%)$ & 6 & 0.398 \\
\hline Management of HIV & $6(85.7 \%)$ & 7 & $2(33.3 \%)$ & 6 & 0.053 \\
\hline Malnutrition & $24(66.6 \%)$ & 36 & $5(88.3 \%)$ & 6 & 0.414 \\
\hline
\end{tabular}

Abbreviation: HIV, human immunodeficiency virus.

treated $75.7 \%$ of cases of diarrhea compared with $84.2 \%$ of cases by nurses in the short training $(P=0.025)$. Nurses in the long training appropriately treated $85.7 \%$ of HIV/acquired immunodeficiency syndrome (AIDS) cases compared with $33.3 \%$ of cases in the short training $(P=0.053)$.

Table 3 displays the results for bivariable and multivariable logistic regression assessing the relationship between the long versus short training on the classification and treatment of six different IMCI illnesses. At the bivariable level and after controlling for confounders at the multivariable level, the only significant differences detected between nurses in the long and short training were the classification of fever

Table 3 Bivariable and multivariable logistic regression to assess the effect of short versus long training on appropriate classification and treatment of childhood illnesses

\begin{tabular}{lll}
\hline Outcomes & \multicolumn{2}{l}{ Short versus long training } \\
\cline { 2 - 3 } & \multicolumn{1}{c}{ OR (95\% CI) } & aOR (95\% CI) \\
\hline Classification/diagnoses & & \\
$\quad$ Pneumonia & $0.97(0.87-1.07)$ & $0.9(0.88-1.09)$ \\
$\quad$ Diarrhea & $0.9(0.81-1.0 I)$ & $0.9(0.80-1.00)$ \\
Fever & $0.7(0.68-0.78)^{*}$ & $0.7(0.64-0.75)^{*}$ \\
Ear infection & $0.9(0.84-1.07)$ & $0.9(0.80-1.05)$ \\
$\quad$ Management of HIV & $1.0(0.98-1.09)$ & $1.0(0.97-1.08)$ \\
Malnutrition & $1.0(0.99-1.11)$ & $1.0(1.00-1.12)$ \\
Treatment/management & & \\
$\quad$ Pneumonia & $0.8(0.68-0.85)^{*}$ & $0.8(0.70-0.89)^{*}$ \\
$\quad$ Diarrhea & $0.9(0.78-1.03)$ & $0.9(0.78-1.07)$ \\
Fever/malaria & $0.9(0.69-1.16)$ & $0.9(0.65-1.16)$ \\
Ear infection & $0.9(0.62-1.30)$ & $0.9(0.64-1.40)$ \\
$\quad$ Management of HIV & $1.5(0.96-2.38)$ & $1.2(0.87-1.86)$ \\
Malnutrition & $0.9(0.59-1.25)$ & $0.8(0.49-1.29)$ \\
\hline
\end{tabular}

Notes: All multivariable analyses control for age, sex, and level of training as confounders. *Statistical significance $P<0.05$.

Abbreviations: aOR, adjusted odds ratio; $\mathrm{Cl}$, confidence interval; $\mathrm{HIV}$, human immunodeficiency virus; OR, odds ratio. (adjusted odds ratio [aOR] 0.7, 95\% confidence interval [CI] $0.64-0.75$ ) and treatment of pneumonia (aOR $0.8,95 \% \mathrm{CI}$ $0.70-0.89$ ). Nurses in the short training had lower odds of inappropriate misclassification and treatment for these two conditions.

\section{Discussion}

The main purpose of this study was to evaluate the effect of shortened IMCI training on appropriate classification and treatment of common childhood illnesses compared with the standard training (long training) using routinely collected data from Rwandan health centers in two districts. The nurses who participated in the long and short training were comparable; however, there was a higher percentage of registered nurses in the short training and a higher percentage of associate nurses in the long training. There was a higher percentage of male nurses in the short training and a higher percentage of female nurses in the long training. Importantly, the groups did not differ significantly on most classifications and treatments of childhood illnesses. The only statistical difference detected was for the classification of fever and the treatment of pneumonia, where nurses in the short training had lower odds of appropriate classification and treatment. This study provides evidence that suggests that a shortened IMCI training does not significantly affect the classification and treatment of childhood illness. In fact, the two differences that were detected illustrated lower odds of misclassification among nurses in the short training. This has important implications for national and international governing bodies and indicates that shorter training has the potential to be cost-effective, have higher program participation, and does not reduce the quality of clinical care. 
Similar results have been demonstrated in other studies. For example, research done in Kosovo found that physicians trained in an 8-day IMCI course performed equally well compared with those trained for the 11-day course. ${ }^{17}$ That study examined health worker performance with management of seven main symptoms and the overall appropriate IMCI classification of under-five children in health facilities. They found no statistically significant difference in the effectiveness of a shortened IMCI training versus the WHO IMCI 11-day standard training.

Our findings are also able to explore the degree of sensitivity for the appropriate diagnosis and treatment of each childhood illness. Within both groups, the illness diagnosed with the highest sensitivity was pneumonia ( $>90 \%)$. In the long training, the illness with the lowest sensitivity was the classification of fever (47\%), and in the short training, it was ear infection $(68 \%)$. This indicates that while we did not detect significant differences between the long and short training, there is still a gap in the correct classification of common childhood illnesses. This likely points to the limited resources available for sensitive and specific diagnoses and not the duration of the training course.

Another interesting finding in this study is that there were many cases and diagnoses of childhood illnesses; however, far fewer records were associated with the treatment and management of each illness. For example, in both the long- and short-training groups, there was a total of 768 diagnoses of HIV; however, there was only 13 cases treated and managed. This highlights a critical gap that is perhaps not identified in the training. In follow-up analyses, we will evaluate the number of diagnoses that go on to get the appropriate treatment, and investigate the reasons for the low level of cases that are treated, and furthermore the cases that receive the appropriate treatment.

Our study findings on HIV management are similar to those from a study done in Zambia on barriers to implementation of the HIV guidelines in the IMCI algorithm, where the study found that $55 \%$ of trained nurses felt that the training in HIV guidelines was inadequate and health workers confirmed that they were uncomfortable with being seen to look through the chart booklet in front of the patients and care-givers. ${ }^{18}$ This resistance to using the chart booklet has been identified in other studies as well. ${ }^{18}$

\section{Limitations}

There are some limitations associated with this study that should be taken into consideration with the interpretation of these results. Due to the secondary nature of the data collected from IMCI medical records, some enrollment errors may have occurred. Additionally, we did not evaluate other factors that could influence nurses' performances, such as supervision, training methodology, health work environment, and direct observation of individual performance. This study utilizes administrative data, which has the benefit of evaluating the actual application and performance of nurses; however, administrative data is always limited by data entry practices and quality. In particular, the use of recorded data to determine the correct diagnosis and treatment of the child is limited when not all of the symptoms and assessment responses have been documented. The ideal study design would evaluate nurses before the training and then randomly assign nurses to either the long or short training. Additionally, two out of 30 districts were selected because they had undergone the IMCI training. Ruhango district was one district that fulfilled inclusion criteria of a shortened IMCI implementation, while Nyanza district was the only district which had not yet received IMCI shortened training. This may affect external generalizability. We encourage further studies to work on all IMCI components in order to show an impact of the IMCI approach to the reduction of under-five mortality.

\section{Conclusion}

IMCI case management training is designed to influence an improvement of quality of care received by underfive children and also health providers' performance in the appropriate management of under-five diseases, also allowing them to catch potential health issues and make a positive impact on the overall health of children under 5 years of age. This study complements similar IMCI evaluations and concludes that there were not major significant differences in clinical classification and treatment of common childhood illness among nurses who completed the long versus short training. The findings of short training seems to address the cost of training and the impact that long training had on health facilities' activities as well as increasing the number of trainees to ensure the appropriate management of illness in under-five children. However, this study illustrates that many cases are still inappropriately classified, and many cases do not go on to receive treatment, whether it is appropriate or inappropriate. This will require follow-up analyses to investigate case management and the operational challenges that may be interfering with appropriate classification and treatment of common childhood illness. In conclusion, the IMCI training can provide an opportunity to emphasize and encourage good quality and immediate individual IMCI 
practices with training, supervision, and a good health workers' environment.

\section{Acknowledgments}

We acknowledge the support of the health center leaders, nurses, and children involved in the study, as well as the IMCI experts: Valens Rurangwa, Claver Mbonabucya, Dieudonne Hakizimana, and Mary Uwimana. We also thank the following individuals for their contribution to data entry: Gerardine Musabyeyezu, Jean de Dieu Mpagazekubwayezu, Samuel Bunane, and Louange Mfurayabo.

\section{Disclosure}

The authors report no conflicts of interest in this work.

\section{References}

1. Rowe AK, Onikpo F, Lama M, Osterholt DM, Rowe SY, Deming MS. A multifaceted intervention to improve health worker adherence to Integrated Management Of Childhood Illness guidelines in Benin. Am J Public Health. 2009;99:837-846.

2. UNICEF Child Mortality Report 2012. Level and Trends in Child Mortality. 32 (2012).

3. Goga AE, Muhe LM, Forsyth K, et al. Results of a multi-country exploratory survey of approaches and methods for IMCI case management training. Health Res Policy Syst. 2009;7:18.

4. Bryce J, Victora CG, Habicht J-P, Black RE, Scherpbier RW. Programmatic pathways to child survival: results of a multi-country evaluation of Integrated Management of Childhood Illness. Health Policy Plan. 2005;20 Suppl 1:i5-i17.

5. Arifeen SE, Bryce J, Gouws E, et al. Quality of care for under-fives in first-level health facilities in one district of Bangladesh. Bull World Health Organ. 2005;83:260-267.

6. Armstrong Schellenberg J, Bryce J, de Savigny D, et al; Tanzania IMCI Multi-Country Evaluation Health Facility Survey Study Group. The effect of Integrated Management of Childhood Illness on observed quality of care of under-fives in rural Tanzania. Health Policy Plan. 2004;19:1-10.
7. Pariyo GW, Gouws E, Bryce J, Burnham G. Improving facility-based care for sick children in Uganda: training is not enough. Health Policy Plan. 2005;20 Suppl 1:i58-i68.

8. Ukwaja KN, Aina OB, Talabi AA. Clinical overlap between malaria and pneumonia: can malaria rapid diagnostic test play a role? $J$ Infect Dev Ctries. 2011;5(3):199-203.

9. Rowe AK, Rowe SY, Holloway KA, Ivanovska V, Muhe L, Lambrechts T. Does shortening the training on Integrated Management of Childhood Illness guidelines reduce its effectiveness? A systematic review. Health Policy Plan. 2012;27:179-193.

10. Karamagi CA, Lubanga RG, Kiguli S, Ekwaru PJ, Heggenhougen K. Health providers' counselling of caregivers in the Integrated Management of Childhood Illness (IMCI) programme in Uganda. Afr Health Sci. 2004;4(1):31-39.

11. Horwood C, Voce A, Vermaak K, Rollins N, Qazi S. Experiences of training and implementation of Integrated Management of Childhood Illness (IMCI) in South Africa: a qualitative evaluation of the IMCI case management training course. BMC Pediatr. 2009;9:62.

12. Goga AE, Muhe LM. Global challenges with scale-up of the Integrated Management of Childhood Illness strategy: results of a multi-country survey. BMC Public Health. 2011;11:503.

13. United Nations. The Millennium Development Goals Report: 2010. New York: United Nations; 2010. Available from: http://www.un.org/ millenniumgoals/pdf/MDG\%20Report\%202010\%20En\%20r15\%20low\%20res\%2020100615\%20-.pdf. Accessed March 23, 2014.

14. National Institute of Statistics of Rwanda, Ministry of Health. Rwanda Demographic and Health Survey; 2010. Available from: http://dhsprogram.com/pubs/pdf/pr7/pr7.pdf.

15. USAID. Improving Child Health in Rwanda: BASICS III; 2009. Available from: http://www.basics.org/reports/FinalReport/Rwanda-FinalReport_BASICS.pdf.

16. Stata | Data Analysis and Statistical Software. at $<$ http://www.stata. $\mathrm{com} />$.

17. Senait kebede, WHO Report of Technical consultation on IMCI training approaches and. 48-15(2007).

18. Mitchell M, et al. Perceived improvement in integrated management of childhood illness implementation through use of mobile technology: qualitative evidence from a pilot study in Tanzania. J Health Commun. 2012;17(Suppl 1):118-27.

\section{Publish your work in this journal}

Risk Management and Healthcare Policy is an international, peerreviewed, open access journal focusing on all aspects of public health, policy, and preventative measures to promote good health and improve morbidity and mortality in the population. The journal welcomes submitted papers covering original research, basic science, clinical \& epidemio-

\section{Dovepress}

logical studies, reviews and evaluations, guidelines, expert opinion and commentary, case reports and extended reports. The manuscript management system is completely online and includes a very quick and fair peerreview system, which is all easy to use. Visit http://www.dovepress.com/ testimonials.php to read real quotes from published authors. 